\title{
Heartbeat: Acute myocardial infarction: one test to diagnose
} them all?

doi:10.1136/heartjnl-2018-313259

The National Institute for Health and Care Excellence (NICE) CG95 Chest Pain of Recent Onset guidelines suggest that in patients presenting with chest pain and a non-diagnostic ECG, a single high-sensitivity troponin (hs-cTn) level below the limit of detection, in combination with a risk stratification tool, be used to identify patients at low risk of myocardial infarction. However, previous studies either used risk scores with limited efficacy or used serial hs-cTn levels below the 99\% percentile. Thus, the efficacy of using a single undetectable hs-cTn test in combination with a low Thrombolysis In Myocardial Infarction (TIMI) score has not been testing.

In an analysis of 3159 adults with chest pain but a non-ischaemic ECG on presentation to the emergency department, Carlton and colleagues ${ }^{1}$ found that the combination of a TIMI score of 0 plus a single negative hs-cTn had a sensitivity of $99 \%$ for the primary outcome of major adverse cardiac events within 30 days. This approach, using an hs-cTn level below the limit of detection as the definition of a negative test, identified $18 \%-21 \%$ of patients at low risk in whom an early discharge strategy would be appropriate (figure 1).

In an accompanying editorial, Chapman and Mills ${ }^{2}$ make the point that this study is the first to validate the NICE guideline using a TIMI score along with a hs-cTn assay below the limits of detection to 'rule-out' myocardial infarction. They also raise the question whether a risk score is needed or whether a simpler approach based on a undetectable hs-cTn level and a normal ECG is reasonable. Both high risk and older patients (TIMI scores over 0 ) also have chronic low-level elevations of hs-cTn levels and thus would not be considered low-risk based on the blood test alone. They caution that 'Comparative studies including risk stratification thresholds alone or in

Correspondence to Professor Catherine M Otto, Division of Cardiology, University of Washington, Seattle, WA 98195, USA; cmotto@uw.edu

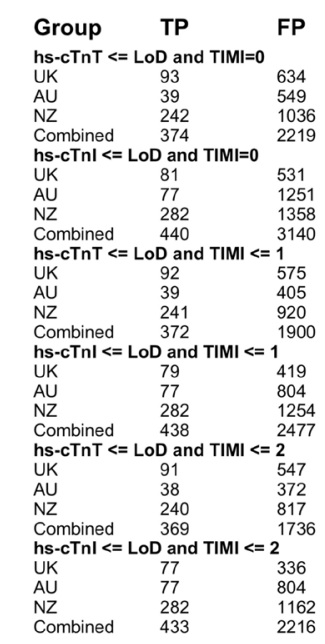

\begin{tabular}{ll} 
FN & TN \\
& \\
1 & 193 \\
1 & 115 \\
0 & 256 \\
2 & 564 \\
2 & \\
2 & 253 \\
3 & 433 \\
0 & 264 \\
5 & 947 \\
2 & 252 \\
2 & 259 \\
1 & 372 \\
1 & 883 \\
4 & \\
4 & 365 \\
3 & 877 \\
3 & 368 \\
7 & 1610 \\
3 & 280 \\
3 & 292 \\
2 & 475 \\
7 & 2783 \\
6 & 448 \\
3 & 80 \\
0 & 460 \\
12 & 1871 \\
\hline
\end{tabular}

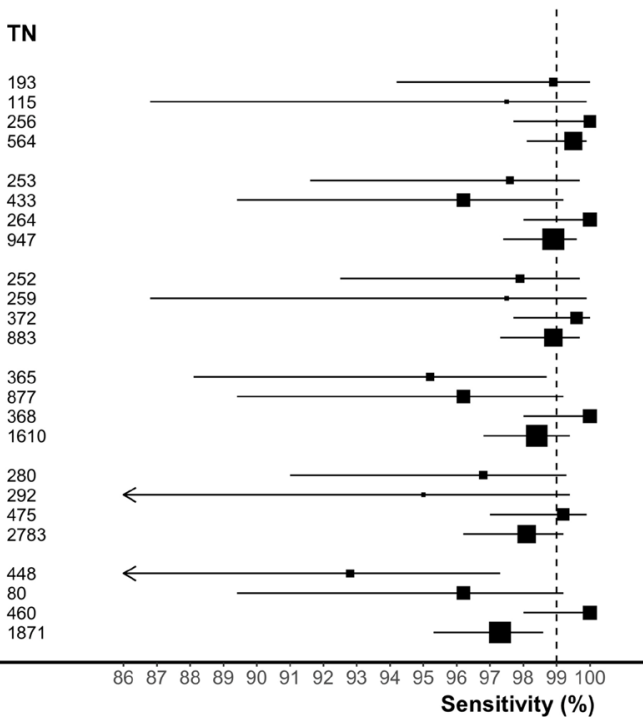

Figure 1 Forest plots of the sensitivity $(95 \% \mathrm{Cl})$ for major adverse cardiac events occurring within 30 days separated by rule-out strategy and study site. FN, false negatives; FP, false positives; LoD, limit of detection; TN, true negatives; TP, true positives; TIMI, Thrombolysis In Myocardial Infarction.

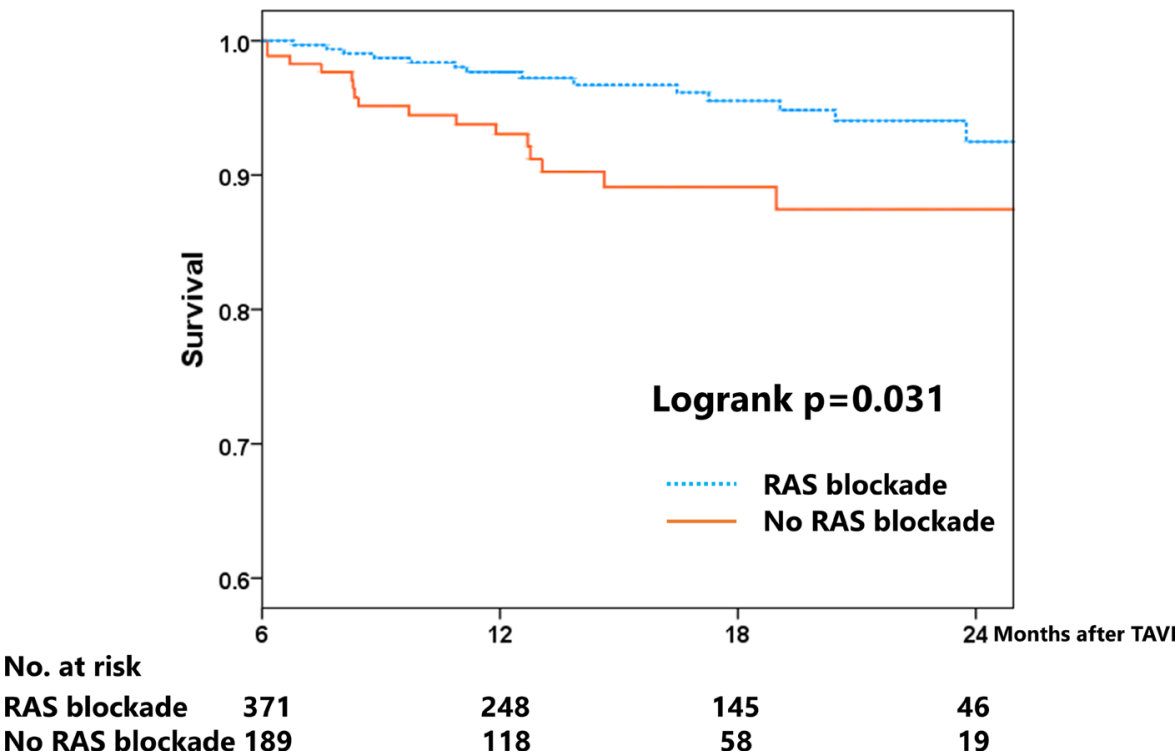

Figure 2 Kaplan-Meier curves of overall survival probability in patients with or without RAS blockade therapy following TAVI. RAS, renin-angiotensin system; TAVI, transcatheter aortic valve implantation.

combination with risk scores are required to determine if improvements in safety can be obtained'. Randomised clinical outcomes studies using the NICE guideline are in progress; in the meanwhile, clinicians should continue to perform serial testing in patients who present early after symptoms onset or with an abnormal ECG and should be aware of the normal reference range of the assay 

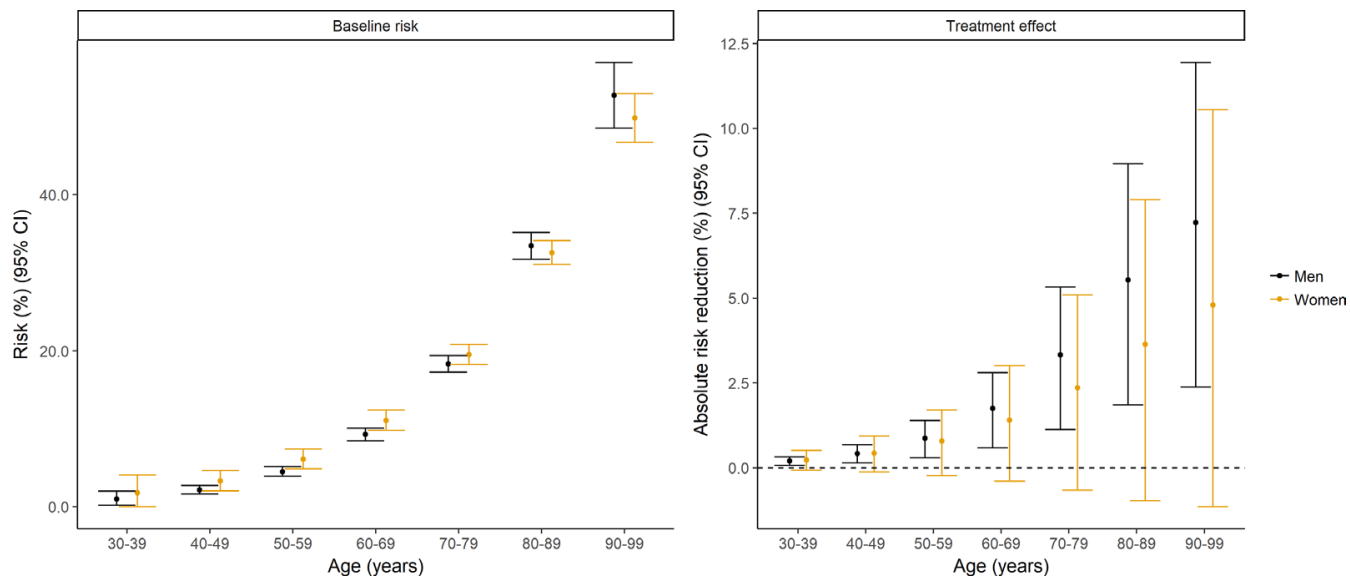

Figure 3 Baseline risk of death and estimates of the absolute treatment effects of oral ${\mathrm{P} 2 \mathrm{Y}_{12}}_{12}$ inhibition in women and men with myocardial infarction in Scotland. Panel A shows estimated risks of deaths obtained from a regression model run on the national Scottish data. A generalised linear model with a log-link and multinomial distribution was fitted to all three outcomes (cardiovascular death, bleeding death and noncardiovascular, non-bleeding death), which included parameters for age and sex as well as age-sex interactions. Panel B shows the modelled absolute risk reduction in this population, obtained from applying to this model the relative treatment efficacy for cardiovascular events and bleeding events in men and women. Most patients in the clinical trial data were aged less than 75 years, and we were unable to examine whether treatment effects showed heterogeneity by age. As such, estimates for older patients should be treated with caution.

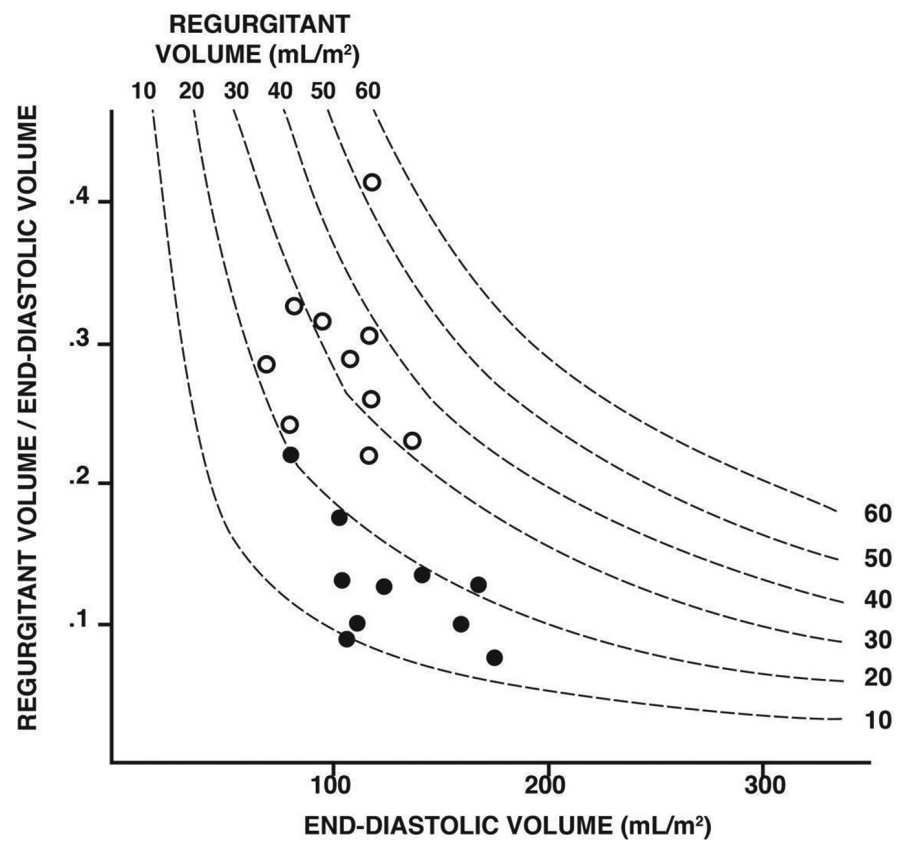

Figure 4 Schema illustrating the relation between the ratio of regurgitant volume to enddiastolic volume (RegV/EDV) and the EDV in patients with mitral regurgitation (MR); the isopleths of RegV are shown as a function of the two variables. Open circles represent average values taken from published studies of primary MR; closed circles represent average values taken from published studies of secondary MR. The triangle, the square and the diamond each represent an average value from the three randomised studies of surgical results in secondary MR. In these studies, the RegV/EDV is low $(0.14,0.16$ and 0.21$)$ and the absolute RegV is less than is seen in most of those with primary MR. This indicates that the effect of surgical correction of secondary MR on the remodelled ventricle would be less than that expected in primary MR. ${ }^{8}$

used at your institution. Finally, 'implementation of the proposed early rule-out strategy should include clinical audit, with attention paid to the time taken to rule out the diagnosis and on the clinical outcomes of patients with suspected acute coronary syndrome'.
Left ventricular (LV) hypertrophy in adults with aortic stenosis (AS) often fails to regress fully after valve replacement and can be associated with persistent diastolic dysfunction and clinical symptoms. Ochiai and colleagues ${ }^{3}$ hypothesised that renin-angiotensin system (RAS) blockade after transcatheter aortic valve implantation (TAVI) for severe AS would be associated with greater LV mass regression and reduced mortality. In a prospective registry of patients undergoing TAVI, there was more LV mass regression at 6 months in the 371 patients who received RAS blockage compared with the 189 patients not on RAS blocking medications. In addition, all-cause mortality was lower in the RAS blockage patients (HR, $0.45 ; 95 \%$ CI 0.22 to $0.91 ; \mathrm{p}=0.025$ ), even after adjusting for confounding variables (figure 2).

Gotzmann ${ }^{4}$ discusses the findings of this small observational study, which suggests that outcomes in adults with severe AS might be improved, even after TAVI, with RAS blockade therapy. LV hypertrophy in adults with AS is presumed to be 'compensatory' to maintain a normal wall stress in response to pressure overload due to outflow obstruction. On the other hand, LV hypertrophy has adverse effects including diastolic dysfunction, increased LV filling pressures and interstitial fibrosis that often persist long after relief of valve obstruction. In addition, many adults with AS also have systemic hypertension, imposing a 'double-load' on the LV. Thus, it seems plausible that RAS blockade would be beneficial, both for reducing blood pressure and improving LV function with previous epidemiological studies supporting this hypothesis. However, 'the study by Ochiai et al is the first to demonstrate the utility of drug therapy after TAVI'. 'The encouraging results, as well as 
the limitations of previous retrospective studies, should prompt us to begin a prospective study to investigate the importance of RAS blockade in patients with AS.'

Despite advances in our understanding of sex differences in cardiovascular disease, women continue to have higher overall annual cardiovascular mortality rates. In meta-analysis and modelling study, Lee and colleagues ${ }^{5}$ found that the higher adverse cardiovascular events rates in women with an acute coronary syndrome were offset by a slightly higher relative risk reduction with the newer oral antiplatelet agents (OAA) ticagrelor and prasugrel compared with clopidogrel. Overall, the absolute risk reduction at 1 year was similar in women and men for cardiovascular mortality $(2.70 \%$ $(95 \%$ CI $-0.63 \%$ to $5.74 \%)$ versus $2.72 \% \quad(95 \%$ CI $0.92 \%$ to $4.35 \%))$, overall morality and bleeding events with these newer agents (Figure 3).

In an editorial, Serebruany and colleagues $^{6}$ cautions us that "all OAA mega trials suffer from massive doubledigit incomplete follow-up rates and/ or drug discontinuations challenging the quality of the analysed datasets and limiting the practical value of further subanalyses mining. Overall, the current knowledge suggests that gender differences should be considered when picking the particular OAA, length of therapy and careful selection of concomitant medications. Whether gender per se is responsible for such differences is still unclear. Further comparative randomised studies of different OAA in patients of both sexes are urgently needed".

A two-part review article on secondary mitral regurgitation (MR) by Gaasch and Meyer ${ }^{78}$ in this issue is essential reading for understanding the pathophysiology, clinical stages, approaches to quantitation of disease severity, and the role in interventional and surgical approaches to improving outcomes in patients with secondary MR.

\section{Competing interests None declared.}

Patient consent Not required.

Provenance and peer review Commissioned; internally peer reviewed.

(C) Article author(s) (or their employer(s) unless otherwise stated in the text of the article) 2018. All rights reserved. No commercial use is permitted unless otherwise expressly granted.
Check for updates

To cite Otto CM. Heart 2018;104:625-627.

Heart 2018;104:625-627.

doi:10.1136/heartjnl-2018-313259

\section{REFERENCES}

1 Carlton EW, Pickering JW, Greenslade J, et al. Assessment of the 2016 National Institute for Health and Care Excellence high-sensitivity troponin rule-out strategy. Heart 2018;104:665-72.

2 Chapman AR, Mills NL. A single blood test to rule out acute coronary syndrome. Heart 2018;104:632-3.

3 Ochiai T, Saito S, Yamanaka F, et al. Renin-angiotensin system blockade therapy after transcatheter aortic valve implantation. Heart 2018;104:644-51.

4 Gotzmann M. Renin-angiotensin system blockade after TAVI: is there a link between regression of left ventricular hypertrophy and prognosis? Heart 2018;104:628-9.

5 Lee KK, Welton N, Shah AS, et al. Differences in relative and absolute effectiveness of oral $P 2 Y_{12}$ inhibition in men and women: a meta-analysis and modelling study. Heart 2018;104:657-64.

6 Serebruany V, Lomakin N, Marciniak T. Meta-analyses of incomplete trial datasets: unreliable and potentially misleading. Heart 2018;104:630-1.

7 Gaasch WH, Meyer TE. Secondary mitral regurgitation (part 1): volumetric quantification and analysis. Heart 2018;104:634-8.

8 Gaasch WH, Meyer TE. Secondary mitral regurgitation (part 2): deliberations on mitral surgery and transcatheter repair. Heart 2018;104:639-43. 